Title

Congenital adrenal hyperplasia (CAH) or Wolff-Parkinson-White (WPW): the difficulties of diagnosing a shocked neonate

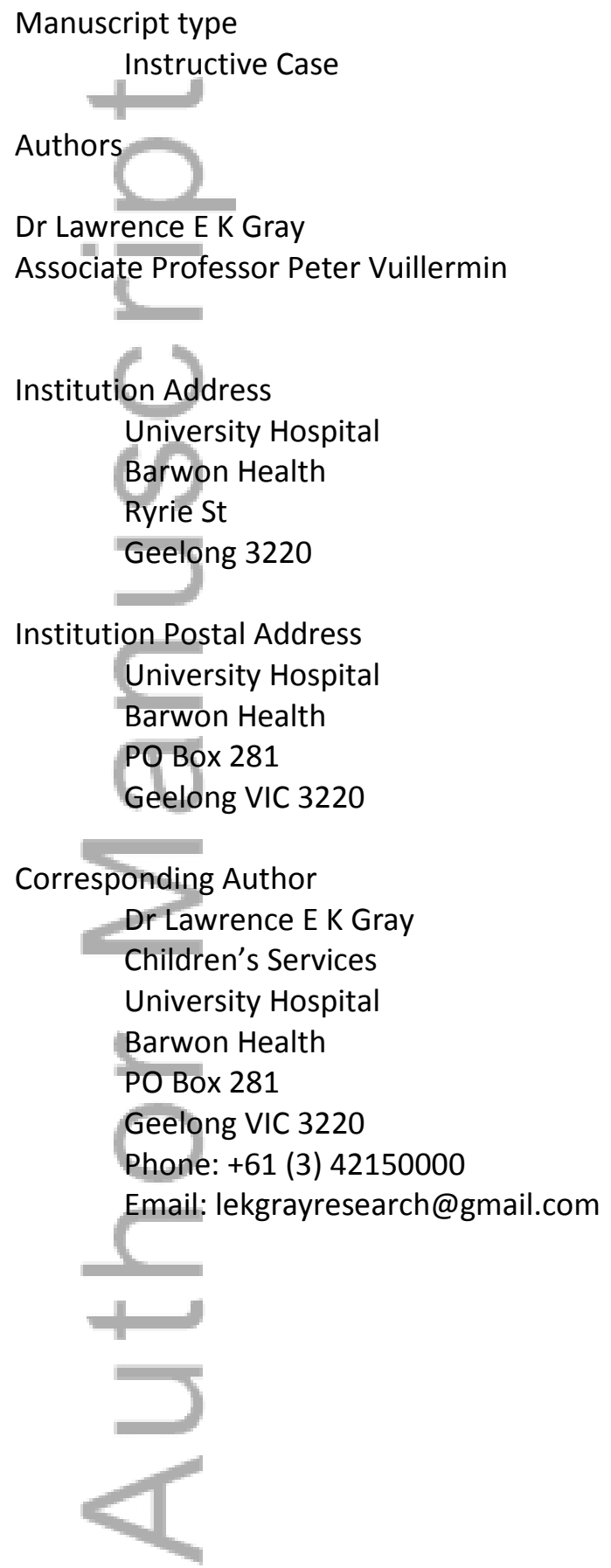

This is the author manuscript accepted for publication and has undergone full peer review but has not been through the copyediting, typesetting, pagination and proofreading process, which may lead to differences between this version and the Version of Record. Please cite this article as doi: 10.1111/jpc.13201

This article is protected by copyright. All rights reserved. 


\section{Instructive Case}

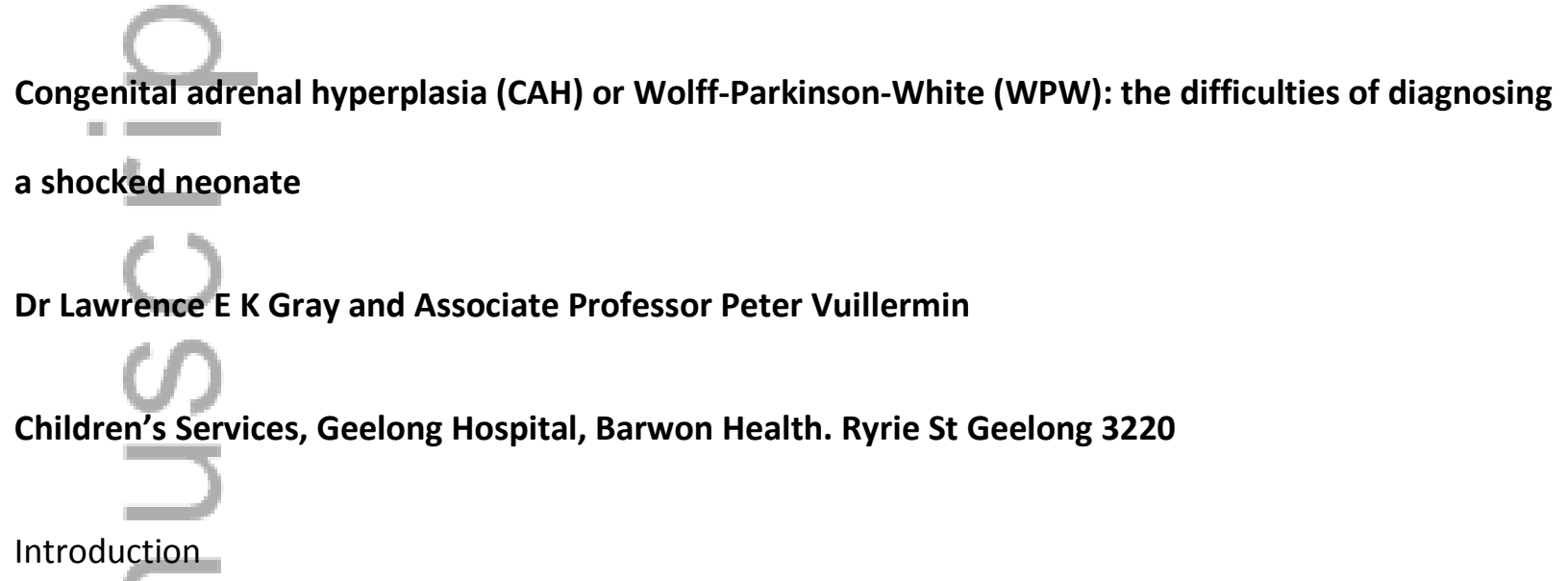

The acutely unwell and shocked neonate is an important clinical challenge for paediatricians. In general, management involves concurrent resuscitation and investigation of the underlying cause. Although the most common causes of shock in the neonatal period are sepsis and dehydration, there are a wide range of other diagnostic possibilities that should be considered.(1) Here we present the case of a neonate who presented with shock, hyponatraemia and hyperkalaemia. The diagnostic journey was challenging and instructive.

\section{Case study}

A 14 day old, ex-38/40 male born by normal vaginal delivery presented to the Emergency Department of our hospital with a three day history of poor feeding and reduced wet nappies. 
On examination he looked unwell, with a mottled grey-bronze colouration, cool peripheries and delayed capillary refill time. His heart rate was 140 and regular, respiratory rate 100 , rectal temperature $33.5^{\circ} \mathrm{C}$ and on cardiovascular examination he was noted to have a gallop rhythm and a 2/6 systolic murmur. On abdominal examination he was noted to have hepatomegaly. The femoral pulses were palpable bilaterally. His scrotum appeared well pigmented (see Figure 1). His genitalia were of normal appearance with both testes present. His weight had increased from a birth weight of $3050 \mathrm{~g}$ to $3560 \mathrm{~g}$. He was initially treated in the emergency department for shock with $20 \mathrm{ml} / \mathrm{kg}$ of intravenous (IV) $0.9 \%$ Saline and IV benzylpenicillin and gentamicin to cover the possibility of sepsis. His blood tests showed a normal full blood examination and C-reactive protein. His urea and creatinine were normal but he was hyponatraemic ( $\mathrm{Na}^{+} 128 \mathrm{mmol} / \mathrm{l}$ Normal Range(NR) 136-148) and hyperkalaemic $\left(\mathrm{K}^{+} 6.3 \mathrm{mmol} / \mathrm{I} \mathrm{NR} 3.4-\right.$ 6.0). His liver function tests were mildly deranged with a GGT of $86 \mathrm{U} / \mathrm{L}(\mathrm{NR}<45), A L T 247 \mathrm{U} / \mathrm{L}(\mathrm{NR}<40)$, AST 233 U/L (NR <35) and Bilirubin 177 micromol/L (NR 1-20). His chest X-ray appeared normal and no abnormality was detected on initial review of his electrocardiogram (ECG).

Given his hyponatraemia, hyperkalaemia and well pigmented scrotum we considered a diagnosis of congenital adrenal hyperplasia (CAH). A further venous blood sample was collected for 17-OH progesterone levels and thyroid function tests, although a serum cortisol was not performed at this time. Urine was also collected for urinary androgens, urinary sodium and a urine metabolic screen. He was then administered $25 \mathrm{mg}(7 \mathrm{mg} / \mathrm{kg})$ of IV hydrocortisone. Following these interventions he improved rapidly and within 2 hours had a significant diuresis and resolution of his systolic murmur and gallop rhythm. He was admitted to the ward and received ongoing hydrocortisone $3 \mathrm{mg}(0.85 \mathrm{mg} / \mathrm{kg}) \mathrm{IV} 4$ hourly. The following morning his repeat blood tests showed a normal full blood examination, urea, 
creatinine and electrolytes and an improvement in liver function tests. An abdominal ultrasound (US) was ordered to investigate for adrenal gland hypertrophy which is usually present in $\mathrm{CAH}$.

However, the abdominal US demonstrated that the adrenal glands were not enlarged. As a result we considered causes of hypoadrenalism other than $\mathrm{CAH}$ that may have responded to fluids and steroid replacement. Results of the initial workup for $\mathrm{CAH}$ were still pending and in light of clinical $=$

improvement, no further investigations were commenced at this time.

Twenty hours after presentation, he became agitated, with a mottled appearance and oxygen saturation monitoring showed a heart rate of 259. ECG obtained at the time showed a supra-ventricular tachycardia (SVT) with a heart rate of 292 (Figure 2). He was initially treated unsuccessfully with vagal manoeuvres, followed by IV adenosine 700 microg $(200 \mathrm{microg} / \mathrm{kg})$. This resulted in an immediate return to sinus rhythm at a rate of $140-150$. Post event ECG demonstrated a delta-wave in the anterior leads, characteristic of Wolff-Parkinson-White Syndrome (WPW) (Figure 3). On review of the initial ECG, subtle evidence of pre-excitation was now noted.

Subsequently, all investigations for $\mathrm{CAH}$ were found to be normal and his hydrocortisone was ceased. He was transferred to a tertiary centre for further cardiac evaluation including echocardiogram and was there commenced on regular atenolol prior to discharge. He presented to our hospital with a further episode of asymptomatic SVT at 1 month of age that was successfully aborted with vagal manoeuvres. He is currently maintained on atenolol and has experienced no further episodes of SVT. Discussion 
The causes of shock can be broadly classified into cardiogenic, distributive, hypovolaemic, obstructive and dissociative.(1) Although this classification is helpful, the priority in shock is to restore delivery of oxygen to tissues and so the first line treatment for all causes remains fluid resuscitation, initially with $0.9 \%$ saline. The history and examination is the key to establishing a likely differential list from this wide range of potential causes, enabling a strategic approach to subsequent investigations. In our case, the examination findings of shock with pigmented genitalia, hyponatraemia and hyperkalaemia suggested a diagnosis of $\mathrm{CAH}$.

$\mathrm{CAH}$ is a group of conditions caused by single or multiple hereditary enzymatic deficiencies. These deficiencies prevent conversion of precursor products to endogenous steroids in the adrenal glands. The most common form of CAH is the 'classic' or salt-wasting 21-hydroxylase deficiency, accounting for $90 \%$ of cases.(2) Overall however, the population incidence of 21-hydroxylase deficiency CAH is relatively uncommon, occurring at a rate of approximately 1 in 16,000 births.(3) In this form of CAH there is typically an absence of both aldosterone (mineralocorticoid activity) and cortisol (glucocorticoid activity). The absence of aldosterone triggers profound renal sodium excretion which causes a secondary dehydration and concentration of the remaining electrolytes. Clinically, this leads to the typical findings of shock, hyponatraemia and hyperkalaemia. The absence of endogenous steroid production also results in an excess of adrenocorticotropic hormone (ACTH) production by the pituitary. In males, this is responsible for hyperpigmentation, whereas in females there may be virilisation of the var.

genitalia at birth. Of relevance for our case, the hyperpigmentation is usually present in both the scrotum and the areolae. Diagnosis is confirmed by a finding of raised serum 17-hydroxyprogesterone (17-OHP) and ACTH levels in association with enlarged adrenal glands on abdominal ultrasound and high 
urinary sodium.(2) Treatment of this presentation of $\mathrm{CAH}$ involves initial resuscitation and administration of IV steroids and then lifetime steroid replacement to substitute both the glucocorticoid and mineralocorticoid functions of the adrenal glands.

Currently CAH caused by 21 hydroxylase deficiency is not included in the Australian newborn screening program (NBS) despite the availability of highly sensitive and specific tests and its use in many $=1$ developed nations. There has been ongoing discussion of the cost effectiveness and benefits of introducing the test to the NBS and small studies examining these questions.(4) There is no doubt that were such a test available, it would have helped in managing our case.

In this case, however, the presentation appears to be the consequence of sustained periods of reduced cardiac output due to intermittent SVT in the context of WPW syndrome. WPW is a condition characterised by an accessory conduction pathway from atria to ventricles. The majority of the time this pathway is anterograde (from atria to ventricles), leading to the characteristic pre-excitation visible on ECG as a delta-wave. When this pathway conducts in a retrograde manner (from ventricles to atria), it can establish a re-entry circuit, looping down through the normal pathway and returning rapidly via the aberrant pathway, leading to supraventricular tachycardia (SVT).(5) The ECG changes required to diagnose WPW characteristically include a delta wave, indicative of ventricular pre-excitation triggered through the accessory pathway, as well as a shortened P-R interval. The incidence of symptomatic WPW in the neonatal period is estimated at $0.1-0.2 \%$ and the risk of associated SVT is high in the neonatal period, with a secondary peak in late childhood.(5) 
It has been noted by some authors that pre-excitation is not a fixed finding in neonatal ECGs and may even remain absent for years after presentations with paroxysmal SVT.(6) In our case, the initial ECG performed at presentation was in sinus rhythm at an age appropriate rate. On close inspection, there is a shortened PR interval and delta wave visible in the anterior leads. These changes were more marked on ECGs taken following the episode of SVT. This is in keeping with a variable degree of detectable preexcitation in neonatal WPW.

WPW carries a $2-4 \%$ lifetime risk of sudden cardiac death secondary to ventricular conduction of an AF or atrial flutter rhythm.(5) However, in the neonatal period these rhythms are rare and the predominant WPW associated tachyarrhythmia is SVT.(7) Prolonged tachycardia in neonates will eventually cause cardiogenic shock, although it is difficult to be certain of the timing and degree of circulatory derangement if the SVT has been intermittent. The mechanism for this is a rate related myocardial hypoxia leading to decreased cardiac output and subsequent cardiac failure. Clinically, this leads to signs of fluid overload, which include gallop rhythm, poor feeding, hepatomegaly and increased work of breathing with activity. Typically, cardiogenic shock in this age group would be accompanied by significant change in myocardial function and the patient may have developed features such as cardiomegaly demonstrable on chest x-ray, however echocardiographic assessment performed at the time of presentation would be more sensitive for this finding.

There are some difficulties in explaining the biochemical derangements found in our case and the rapidity of the resolution, as they would not be typically routinely found in an infant with cardiogenic shock. Cardiac failure triggers activation of the renin-angiotensin system, prompting sodium and fluid retention. However, as even well neonates are prone to significant renal sodium loss, the combination 
of fluid overload and poorly perfused and immature kidneys may have affected the maintenance of electrolytes, leading to the observed mild hyponatraemia. Hyperkalaemia could be caused by renal dysfunction but may also have a simple explanation such as a haemolysed specimen in a difficult to obtain blood sample from a shocked patient.

Our case demonstrates that appreciating subtle findings requires a broad differential diagnosis which $=$

must be maintained even in the face of a clever working theory. Only then will the delta waves on the initial ECG (or other subtle findings) declare themselves!

1. McKiernan CA, Lieberman MD. Circulatory Shock in Children. Pediatr Rev. 2005;26(12):451.

2. Antal Z, Zhou P. Congenital Adrenal Hyperplasia: Diagnosis, Evaluation, and Management. Pediatr Rev. 2009 Jul 1;30(7):e49-57.

3. Speiser PW, White PC. Congenital adrenal hyperplasia. N Engl J Med. 2003;349(8):776-88.

4. Wu JY, Sudeep, Cowley DM, Harris M, McGown IN, Cotterill AM. Is it time to commence newborn screening for congenital adrenal hyperplasia in Australia? Med J Aust. 2011 Sep 5;195(5):260-2.

5. Salerno JC, Seslar SP. Supraventricular tachycardia. Arch Pediatr Adolesc Med. 2009;163(3):268-74.

6. Schwartz P. Guidelines for the interpretation of the neonatal electrocardiogram. Eur Heart J. 2002 Sep 1;23(17):1329-44.

7. Kothari DS. Neonatal tachycardias: an update. Arch Dis Child - Fetal Neonatal Ed. 2006 Mar 1;91(2):F136-44.

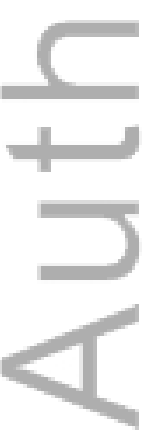

This article is protected by copyright. All rights reserved. 


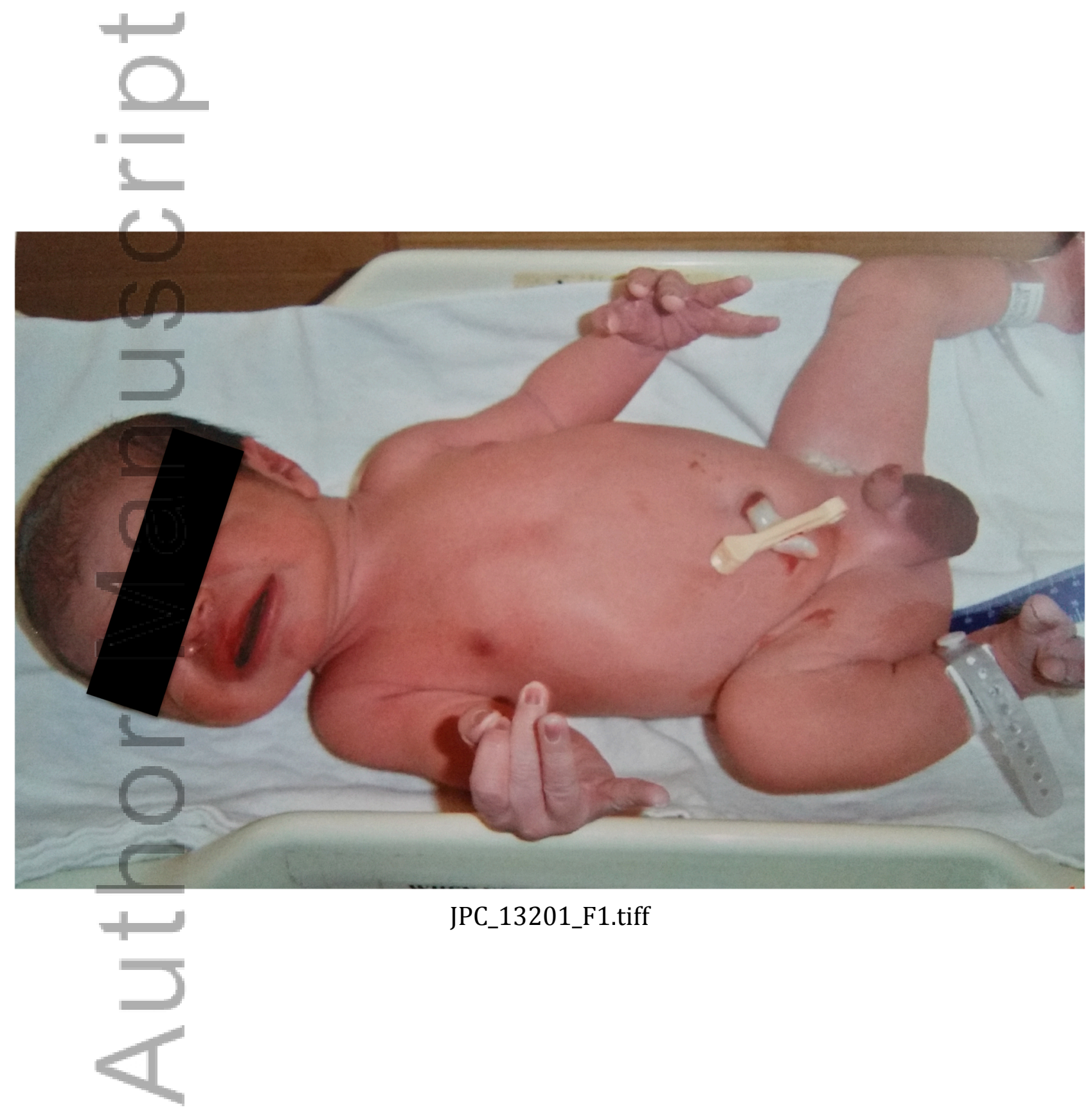

This article is protected by copyright. All rights reserved. 


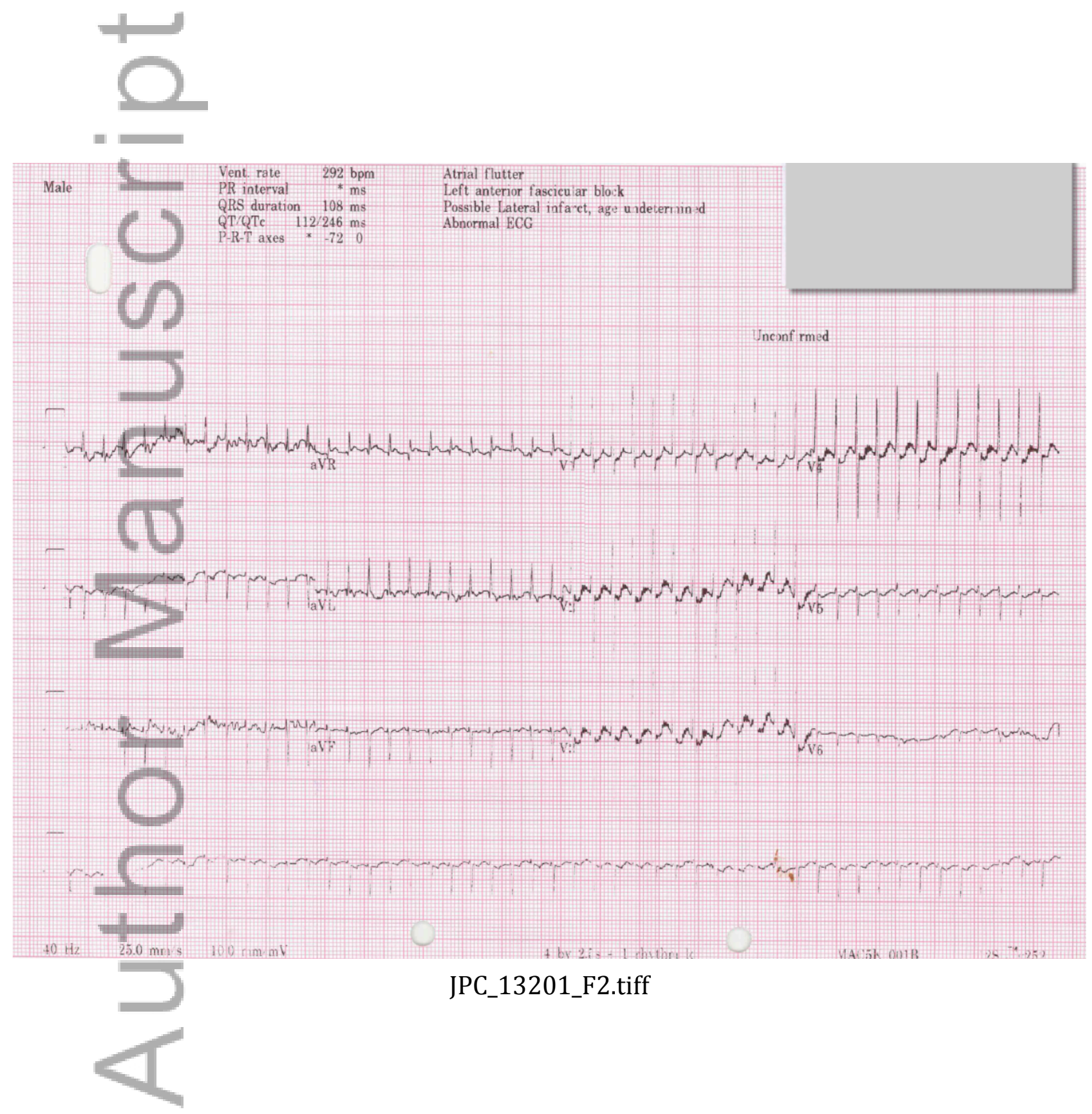

This article is protected by copyright. All rights reserved. 


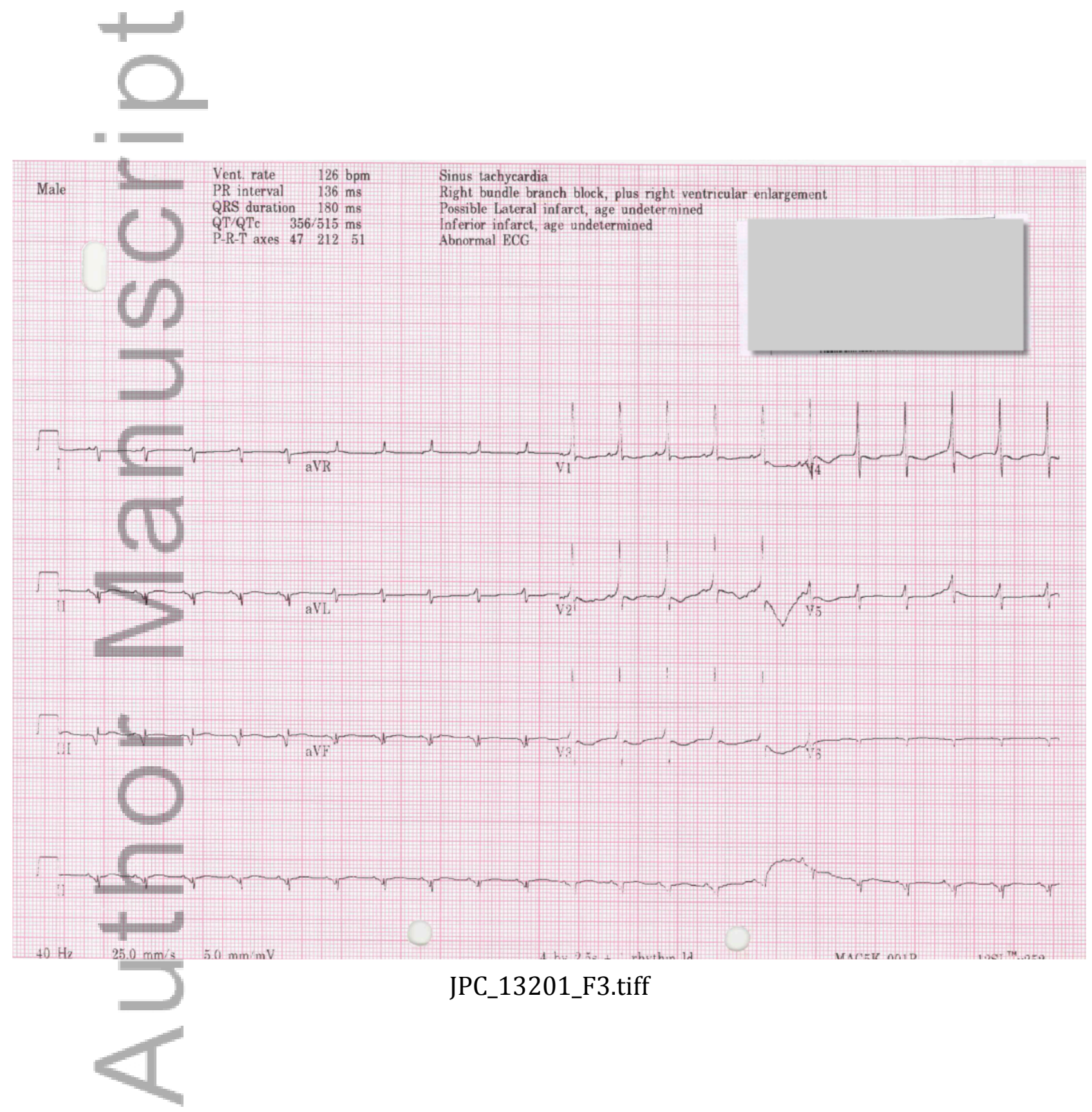

This article is protected by copyright. All rights reserved. 
Learning points

-A shocked neonate requires prompt treatment before diagnosis.

-CAH is not included on newborn screening tests currently.

-CAH caused by 21-hydroxylase deficiency is diagnosed by raised serum 17-

hydroxyprogesterone (17-OHP) and ACTH levels, in association with enlarged adrenal glands on abdominal ultrsound and high urinary sodium.

-WPW in neonates Is likely to present as SVT, however the typical ECG finding of preexcitation may be absent.

-Prôlonged or recurrent SVT leads to cardiac failure in the neonate if untreated.

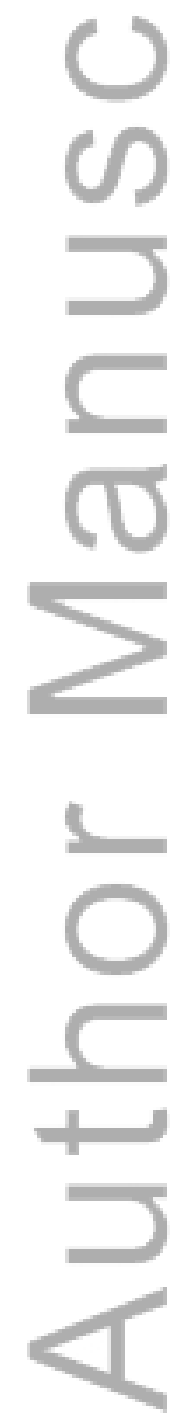

This article is protected by copyright. All rights reserved. 